\title{
Psoríase na infância e na adolescência *
}

\author{
Psoriasis in childhood and adolescence
}

Ricardo Romiti ${ }^{1}$
Marcelo Arnone

\author{
Luciana Maragno ${ }^{2}$ \\ Maria Denise Fonseca Takahashi ${ }^{4}$
}

\begin{abstract}
Resumo: A psoríase é doença inflamatória crônica, imunologicamente mediada, recorrente e de caráter universal. Aproximadamente um terço dos adultos acometidos refere início da doença antes dos 16 anos de idade. Quanto mais precoce, mais grave tende a ser a evolução do quadro. Em crianças, as lesões podem ser fisicamente desfigurantes, causando prejuízos psicológicos e evidente comprometimento da qualidade de vida. As medicações sistêmicas utilizadas na psoríase, bem como a fototerapia, têm indicação limitada na infância, devido aos efeitos cumulativos das drogas, à baixa aceitação e ao risco de teratogenicidade. Nesta seção, discutiremos as principais manifestações clínicas da psoríase na infância e na adolescência, bem como os diagnósticos diferenciais, opções terapêuticas e prognóstico.

Palavras-chave: Adolescente; Criança; Epidemiologia; Infância; Psoríase; Quadro clínico; Terapêutica

Abstract: Psoriasis is a chronic, immunologically mediated, recurrent and universal inflammatory disorder. Approximately one third of adults refer onset before 16 years of age. The sooner the onset, the worse is the prognosis. In children, lesions may be physically disfiguring, leading to psychological impairment and evident loss of quality of life. Systemic therapy used in psoriasis, as well as phototherapy, has limited use in children due to accumulative effects of drugs, low acceptance, and risk of teratogenicity. In this section, we discuss the main clinical aspects of psoriasis in childhood and adolescence, differential diagnosis, therapeutic options, and prognosis.

Keywords: Adolescent; Child; Clinical features; Epidemiology; Infancy; Psoriasis; Therapeutics
\end{abstract}

\section{INTRODUÇÃO}

A psoríase vulgar representa uma dermatose rara na infância e corresponde a cerca de $4 \%$ de todas as dermatoses observadas em doentes menores de 16 anos. ${ }^{1}$ A psoríase de início na infância tem alta incidência familiar. A apresentação mais comum na primeira infância se caracteriza pelo surgimento de placas eritematosas bem delimitadas envolvendo a genitália e as regiões glútea e periumbilical, tendendo a ser persistente e rebelde ao tratamento (Figura 1). O acometimento facial não é raro. Com o passar do tempo, novas placas eritematoescamosas tendem a surgir, acometendo, principalmente, o tronco e os membros.
Variantes da psoríase de início na infância incluem as de acometimento periungueal, com graus variáveis de onicodistrofia (Figura 2), bem como formas restritas ao couro cabeludo. A psoríase gutata é raramente observada em crianças com menos de cinco anos de idade.

O tratamento dependerá, basicamente, da gravidade do quadro, da associação com quadro articular, de eventuais comorbidades, da idade do doente, de terapêuticas prévias e de eventos adversos ocorridos. Ressalta-se que, até o momento, nenhum tratamento sistêmico está autorizado pelo Food and Drug Administration (FDA) para o tratamento da psoríase na

Aprovado pelo Conselho Editorial e aceito para publicação em 04.11.2008.

* Trabalho realizado no Departamento de Dermatologia do Hospital das Clínicas da Universidade de São Paulo (USP) - São Paulo (SP), Brasil Conflito de interesse: Nenhum / Conflict of interest: None

Suporte financeiro: Nenhum / Financial funding: None

Médico assistente. Departamento de Dermatologia do Hospital das Clínicas da Universidade de São Paulo (USP) - São Paulo (SP), Brasil. Médica residente. Departamento de Dermatologia do Hospital das Clínicas da Universidade de São Paulo (USP) - São Paulo (SP), Brasil.

Médico assistente. Departamento de Dermatologia do Hospital das Clínicas da Universidade de São Paulo (USP) - São Paulo (SP), Brasil.

Médica assistente. Departamento de Dermatologia do Hospital das Clínicas da Universidade de São Paulo (USP) - São Paulo (SP), Brasil 
infância. No Brasil, a acitretina e a ciclosporina são indicadas para uso pediátrico no tratamento de casos graves de psoríase. No caso do metotrexato, a bula não especifica indicação para crianças com psoríase, mas refere o seu uso no controle sintomático da psoríase recalcitrante, grave e incapacitante e que não responda adequadamente a outras formas de terapia.

\section{HISTÓRICO}

O primeiro relato histórico da psoríase se deve a Celsus (25 a.C. -45 d.C.). Hipócrates (460-375 a.C.) descreveu lesões de aspecto semelhante à psoríase que classificou como "erupções escamosas", denominando-as lopoi (de lepo, descamar). Foi Galeno (133-200 d.C.) quem cunhou a palavra psoríase, do grego psora, prurido. No entanto, o acometimento palpebral associado a outras lesões psoriasiformes, bem como a presença de descamação e prurido nessa descrição original de Galeno, sugerem a hipótese de que seu relato se referia, na verdade, ao eczema seborreico.

Até o final do século XVIII, psoríase e hanseníase eram classificadas em conjunto e os doentes acometidos eram tratados com o mesmo preconceito e marginalização por parte da sociedade. Coube a Willan, no início do século XIX, a caracterização criteriosa e precisa da psoríase, bem como a descrição de suas diferentes variantes clínicas. Somente em 1841, a psoríase foi definitivamente separada da hanseníase por Ferdinand von Hebra.

\section{EPIDEMIOLOGIA}

Embora a psoríase seja uma dermatose pouco relatada em crianças, a verdadeira prevalência nessa faixa etária é desconhecida. ${ }^{2}$ Estima-se que de $25-45 \%$ dos casos possam iniciar seu curso antes dos 16 anos de idade e, em cerca de $2 \%$ dos casos, antes dos dois anos de vida. ${ }^{3} \mathrm{~A}$ psoríase pode ainda, excepcionalmente, ser congênita ou nevóide. ${ }^{4}$ Embora, no passado, tenha sido observada

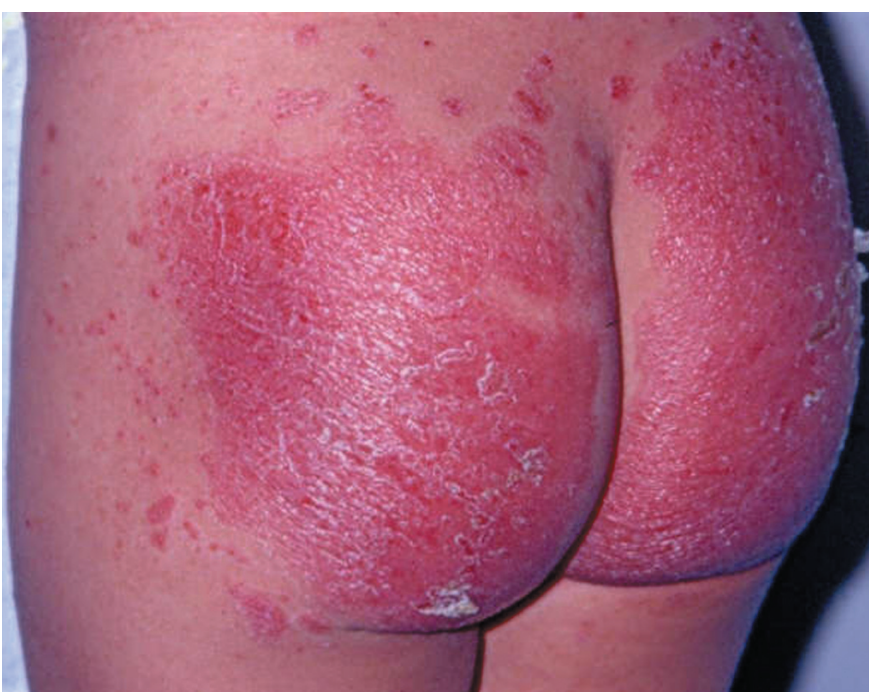

Figura 1: Acometimento isolado da região das fraldas maior prevalência de psoríase em crianças do sexo feminino, estudos atuais indicam que ambos os gêneros são afetados igualmente, como ocorre nos adultos. ${ }^{5,6}$

O risco de desenvolver psoríase é maior quando um dos pais é afetado ou ambos o são. Entre os doentes que desenvolvem psoríase na infância, $49 \%$ apresentam familiares de primeiro grau afetados pela doença, enquanto que, nos doentes com início das lesões na vida adulta, esse número atinge $37 \%$. Estudos com pares de gêmeos demonstram uma concordância entre gêmeos monozigóticos de até $75 \% 6$.

\section{ETIOPATOGENIA}

Apesar dos avanços na última década, a causa da psoríase permanece desconhecida. Trata-se de doença inflamatória crônica da pele e das articulações, imunomediada, com predisposição poligênica, caracterizada por complexas alterações no crescimento e por diferenciação epidérmica e múltiplas anormalidades bioquímicas, imunológicas e vasculares, além da relação não compreendida com os agravos emocionais. No passado, considerava-se o distúrbio dos queratinócitos como a base etiopatogênica da psoríase; entretanto, atualmente, sabe-se tratar de alteração inicialmente imunológica, mediada pela resposta tipo Th1. , $^{, 7,8,9,10}$

O componente genético envolvido na etiologia da psoríase pode ser evidenciado por estudos sobre a incidência familiar, incidência de casos na prole, grau de concordância entre gêmeos e identidade de antígenos de histocompatibilidade (HLA). Múltiplos alelos HLAs têm sido associados à psoríase, especialmente: HLACw6, HLA-B13, HLA-B17, HLA-B37, HLA-DR7, HLA-B46, HLA-B57, HLA-Cw1 e HLA-DQ9. Estudos recentes revelam loci de susceptibilidade denominados Psors, localizados nos cromossomos 6p, 17q, 4q e 1q. ${ }^{5,6,11}$

Estudos populacionais com gêmeos monozigóticos demonstram haver, também, a participação de fato-

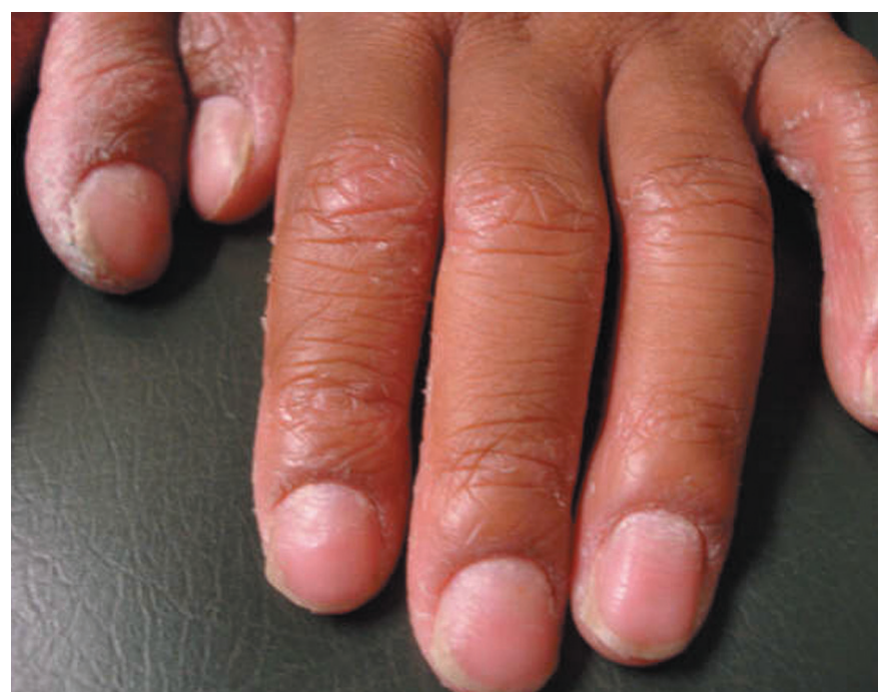

FIGURA 2: Onicodistrofia associada à psoríase 
res ambientais no processo, entre eles, trauma cutâneo, infecções (estreptococo Beta-hemolítico, HIV), drogas (lítio, betabloqueadores, antimaláricos e interrupção de corticoterapia - podendo, neste caso, levar à grave psoríase pustulosa generalizada e à eritrodermia), vacinação, fatores psicogênicos/emocionais, distúrbios endócrinos e metabólicos, tabagismo, abuso de álcool e variações climáticas. ${ }^{12}$ A possível exacerbação da psoríase pelo uso de anti-inflamatórios não hormonais, tetraciclinas e inibidores da ECA deve ser vista com cautela, pois, apesar da alta frequência da utilização dessas medicações, esparsos são os relatos que associam seu uso à piora da psoríase. $^{13}$

Na patogenia, há uma aceleração do ciclo germinativo epidérmico, um aumento das células em proliferação e um encurtamento do tempo de renovação celular na epiderme tanto da lesão quanto na pele "normal" do paciente com psoríase. Acredita-se que essa hiperproliferação de queratinócitos se deva a um aumento da quantidade de fator de crescimento epidérmico (EGF), de fator de crescimento e transformação alfa (TGF-alfa) e da participação de citocinas pró- inflamatórias (IL-1, IL6, IFN-gama), que atuariam como mitógenos para essas células. Outro mecanismo implicado seria a falha na resposta dos queratinócitos presentes nas lesões de psoríase a citoquinas inibitórias (IFN $\gamma, \mathrm{TNF} \alpha, \mathrm{TGF} \beta$ ) produzidas por linfócitos CD8. Para a psoríase do tipo "em gota", também se descreve a autoimunidade induzida por reação cruzada a antígenos estreptocócicos.

\section{ASPECTOS CLÍNICOS}

A frequência relativa dos tipos clínicos de psoríase e as formas de apresentação da doença diferem entre os adultos e as crianças. A psoríase em placas é a variante clínica mais comum em crianças e adolescentes (34$84 \%$ ), desconsiderando-se a forma que acomete a área das fraldas (psoriatic diaper rash). ${ }^{5}$

As lesões se caracterizam por pápulas e placas eritematosas, bem delimitadas, de tamanhos variados e com descamação prateada, dispostas, não raro, de maneira simétrica. Na infância, a psoríase pode apresentar características atípicas, ou seja, placas eritematosas únicas ou pouco numerosas e ligeiramente descamativas, acometendo áreas insólitas, especialmente a região da face - incluindo a porção periorbitária, perioral e nasal - e dificultando o diagnóstico, muitas vezes (Figura 3). Característico da psoríase infantil é o acometimento folicular com prurido variável, mais bem detectado nas lesões dos membros (Figura 4A). ${ }^{14,15,16}$ Formas leves simulando pitiríase alba podem ocorrer (Figura 4B).

As lesões de psoríase acometem, em geral, o couro cabeludo, seguido da superfície extensora das extremidades e tronco. Ocorre, frequentemente, distribuição simétrica das lesões e ausência de prurido (Figura 5A). O acometimento das mãos, dos pés, da genitália e das áreas flexurais (Figura 5B), inclusive perium- bilical, também é mais comum em crianças. ${ }^{6,17}$

A forma congênita, definida como a ocorrência de qualquer uma das variantes clínicas da psoríase ao nascimento ou durante os primeiros dias de vida, é extremamente rara. Habitualmente, expressa-se na forma de psoríase em placas. Casos de psoríase eritrodérmica congênita ou neonatal são ainda mais raros, são graves e demandam pronta intervenção. $\mathrm{O}$ diagnóstico diferencial, nesses casos, inclui a síndrome da pele escaldada estafilocócica, a síndrome do choque tóxico, a candidíase, as ictioses congênitas, as imunodeficiências como a síndrome de Omenn, os distúrbios metabólicos, a dermatite atópica e seborreica, a pitiríase rubra pilar e a mastocitose generalizada. ${ }^{1}$

O acometimento do couro cabeludo, com a presença de escamas brancas, aderentes e espessas ao redor dos folículos pilosos com leve eritema (pseudotinea amiantacea), pode levar à queda temporária dos cabelos ou mesmo à alopecia psoriásica. Pode ocorrer lesão única em placa ou mesmo lesões mal delimitadas e descamativas, clinicamente indistinguíveis da dermatite seborreica. ${ }^{17,18}$

Topografia característica em crianças é o acometimento na área das fraldas, que ocorre em crianças com até dois anos de idade. Diferentemente da dermatite de fraldas (dermatite de contato), as lesões apresentam eritema mais claro e mais brilhante, bordas bem delimitadas e envolvimento das dobras inguinais, com prurido variável. Classicamente, tais sinais e sintomas respondem muito pouco ao tratamento convencional para dermatite de fraldas. Após uma a duas semanas do aparecimento do eritema na área das fraldas, algumas crianças desenvolvem lesões clássicas de psoríase na face, no couro cabeludo, no tronco e nos membros. ${ }^{17}$

A psoríase gutata é uma variante clínica da psoríase que acomete doentes pediátricos em uma frequência que varia entre $6,4 \%$ e $44 \% 6$. O quadro cutâneo aparece

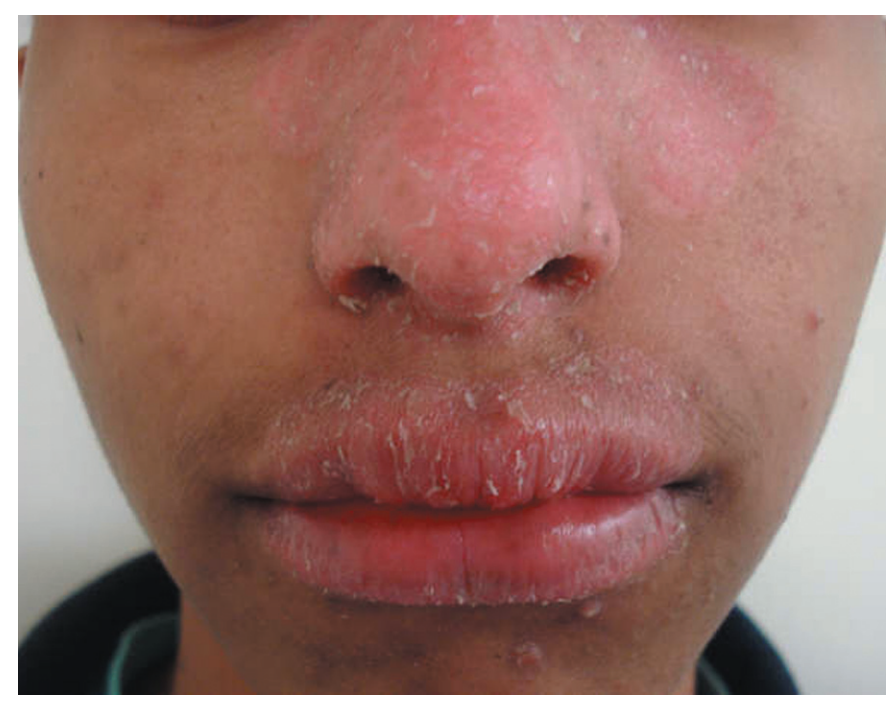

Figura 3: Acometimento centrofacial na psoríase 

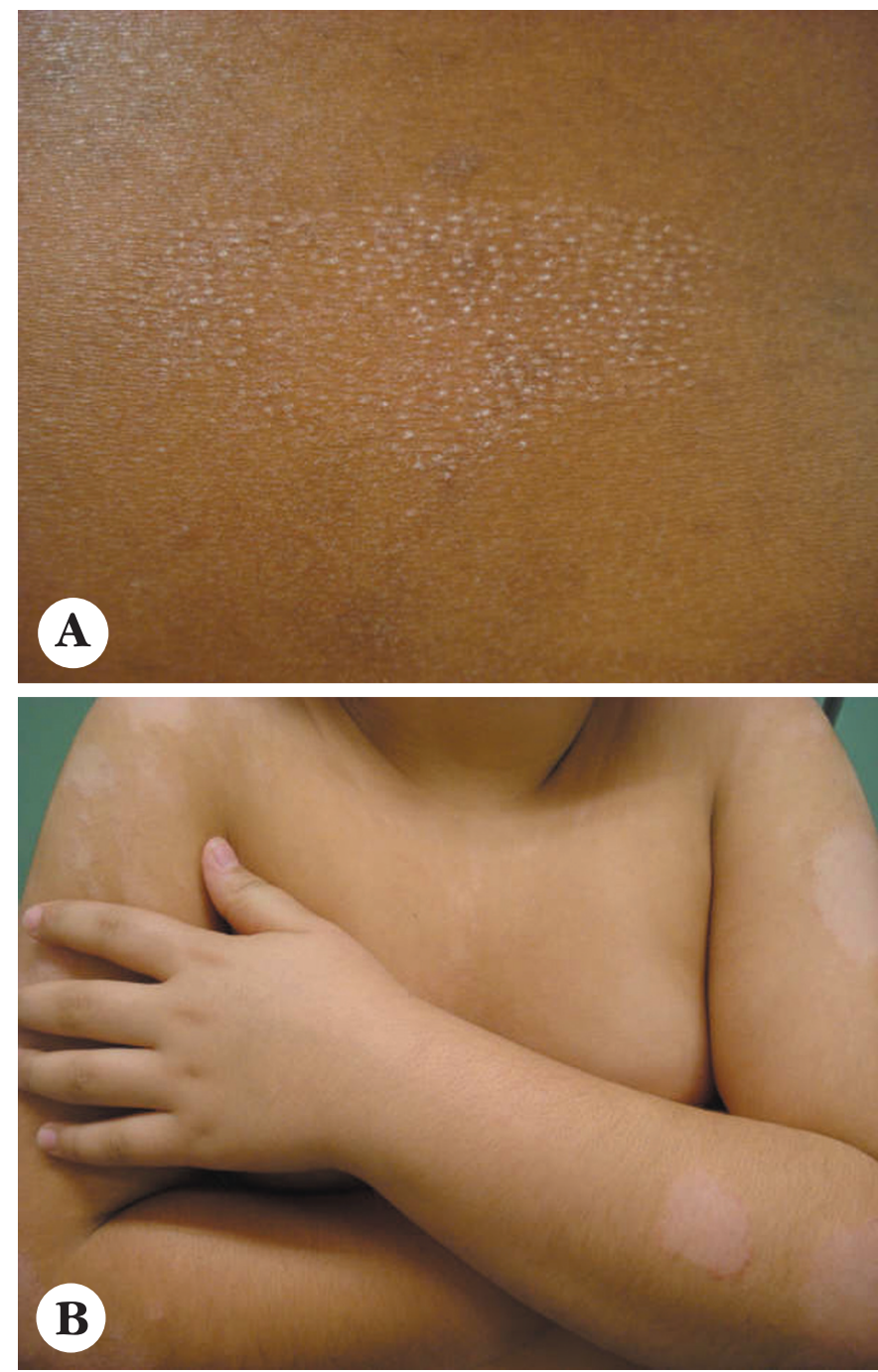

Figura 4: A. Psoríase de padrão folicular; B. Psoríase leve simulando pitiríase alba

de forma abrupta e é precedido, geralmente, por uma infecção estreptocócica (56-85\% dos casos), ${ }^{5}$ comumente de vias aéreas superiores. Lesões papulosas de até $1 \mathrm{~cm}$ de diâmetro estão dispostas simetricamente em toda a superfície corpórea, predominando no tronco e na raiz dos membros. Em geral, a psoríase gutata regride espontaneamente em três a quatro meses. Ocasionalmente, as lesões podem persistir e aumentar de tamanho, tomando as características da psoríase em placas. ${ }^{19}$ Em um prazo de dez anos, entre um e dois terços dos doentes com diagnóstico de psoríase gutata evolui para a forma crônica em placas.

A psoríase linear, forma rara de psoríase, caracteriza-se por lesões eritemato-escamosas seguindo as linhas de Blaschko. Pode ter início na infância ou na idade adulta e acometer, essencialmente, tronco ou membros, com extensão e progressão variáveis. Deve ser diferenciada do nevo epidérmico verrucoso inflamatório linear (Nevil), que surge na infância e acomete, principalmente, as regióes inguino-crural e genital, seguindo também as linhas de Blaschko. O prurido pode ser intenso. Apesar de a histopatologia evidenciar dermatite psoriasiforme, a cronicidade das lesões e a resistência a qualquer forma de intervenção terapêutica o diferenciam da psoríase linear. ${ }^{6,20}$

A psoríase pustulosa é rara em crianças. Caracteriza-se por múltiplas pústulas, estéreis, sobre base eritematosa. Pode ser generalizada ou localizada. A forma generalizada (von Zumbusch) pode ser desencadeada em um paciente com psoríase vulgar por interrupção do corticóide sistêmico, por hipocalcemia, por infecção ou por irritantes locais. Geralmente, há comprometimento do estado geral, febre alta e leucocitose. A erupção é súbita e generalizada, porém, comumente, persiste por poucas semanas, revertendo ao quadro anterior ou transformando-se em psoríase eritrodérmica (Figura 6). A forma localizada compreende três subformas: psoríase pustulosa em placas ou anular, acrodermatite contínua de Hallopeau (pústulas ou lagos de pus nos quirodáctilos e, mais raramente, dos pododáctilos, de curso crônico, sem tendência a remissão espontânea) e pustulose palmoplantar (caracterizada por surtos de pústulas estéreis que comprometem palmas e/ou plantas, simetricamente, sem manifestações). ${ }^{19}$ Complicações raras dessa forma de psoríase, descritas em crianças, são insuficiência renal, icterícia colestática, lesões líticas ósseas e osteomielite multifocal estéril. ${ }^{1} \mathrm{~A}$ forma anular, que associa lesões eritematodescamativas e pústulas periféricas, parece ser exclusiva da infância (Figura 7). ${ }^{2}$ Essa variante pode ser acompanhada de febre, osteomielite asséptica e comprometimento pulmonar.

Apresentações clínicas menos frequentes nas crianças, quando comparadas aos adultos, são psoríase eritrodérmica e artrite psoriática. Na psoríase eritrodérmica, ocorre eritema intenso, universal, com descamação variável. Pode acontecer na evolução natural da doença ou, mais comumente, após terapias intempestivas, interrupção de corticoterapia sistêmica ou em pacientes com Sida. Há nítido predomínio do eritema sobre a descamação. Pode ocorrer hipertermia ou hipotermia e, em casos de longa duração, pode haver diminuição do débito cardíaco e comprometimento das funções hepática e renal. $\mathrm{O}$ iminente risco de choque cardiovascular e mesmo séptico torna esses doentes extremamente graves, demandando imediata internação e pronta intervenção terapêutica associada a medidas de suporte..$^{1,16,21}$

A artrite psoriásica caracteriza-se por uma forma de artrite soronegativa, encontrada entre $5 \%$ e $42 \%$ dos doentes de psoríase, sendo rara na infância. ${ }^{22}$ Por outro lado, 8-20\% dos casos de artrite na infância são diagnosticados como artrite psoriática6. O pico de incidência está entre 9 e 12 anos de idade e o sexo feminino é ligeiramente mais acometido ( $\mathrm{F}: \mathrm{M}=3: 2) 1$. Eventualmente, alterações oftalmológicas podem estar associadas à artrite psoriásica.

A forma mais frequente de artrite psoriásica é a 

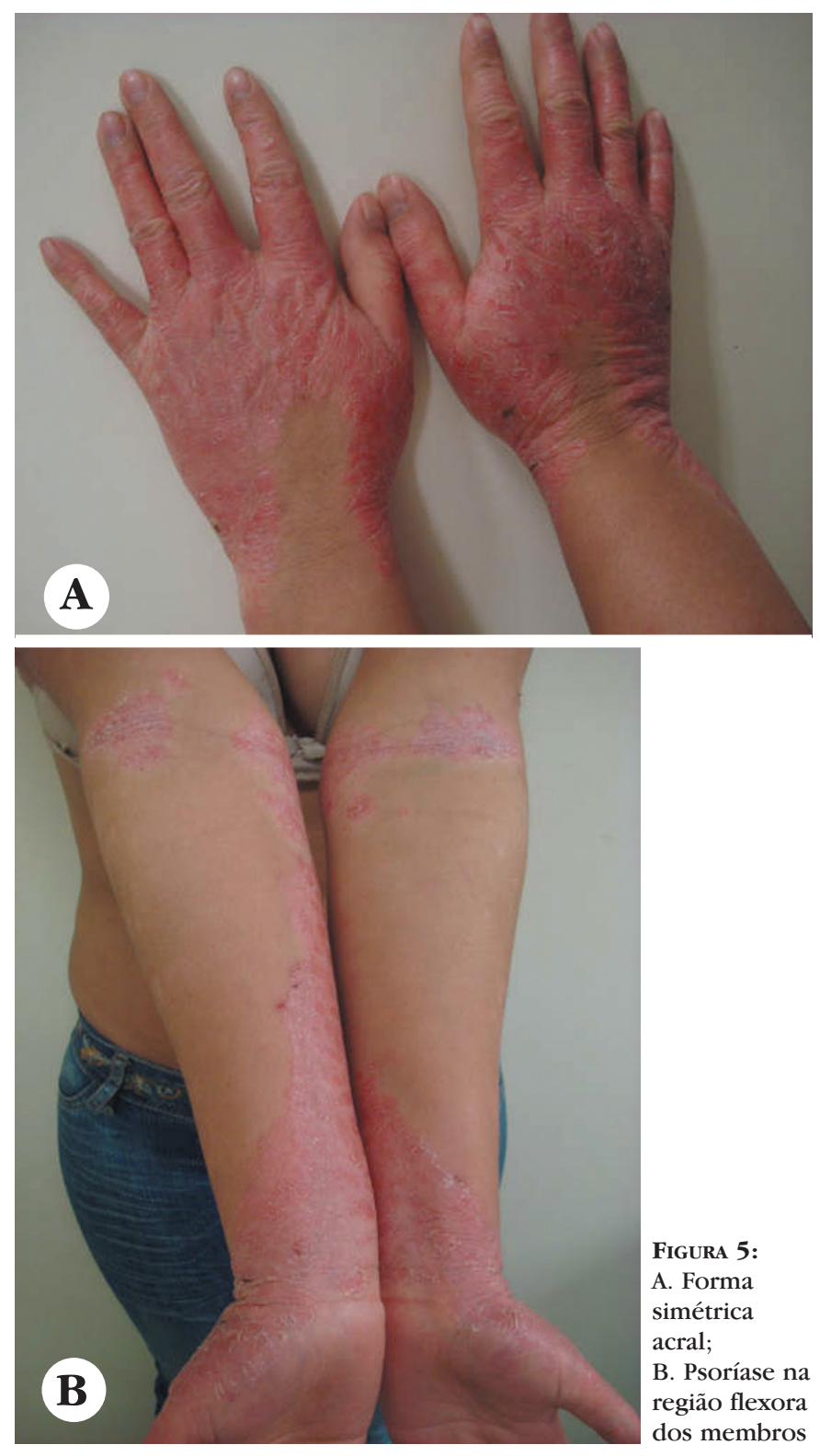

Figura 5:

A. Forma simétrica acral;

B. Psoríase na região flexora dos membros

monoartrite ou oligoartrite assimétrica, que afeta, principalmente, articulações das mãos e dos pés. Outras formas menos comuns apresentam comprometimento simétrico, axial e, até mesmo, mutilante. A artrite pode preceder (19\%) o quadro cutâneo, aparecer concomitantemente (16\%) ou surgir depois (em média, dez anos) do início da psoríase na pele $(65 \%)$. Quase todas as formas de psoríase podem cursar com artrite; em geral, quanto mais grave o quadro cutâneo, maior a prevalência da artrite. Os quadros cutâneo e articular não têm relação do ponto de vista de atividade e evolução. A uveíte psoriásica, uma forma de uveíte anterior assimétrica, ocorre em 14-17\% de crianças com artrite psoriásica juvenil. ${ }^{22,23,24}$

Alterações ungueais são observadas entre 10\% e $40 \%$ das crianças com psoríase, podendo preceder o aparecimento das lesões cutâneas e ser, durante anos, a única manifestação da afecção. O grau de envolvimento

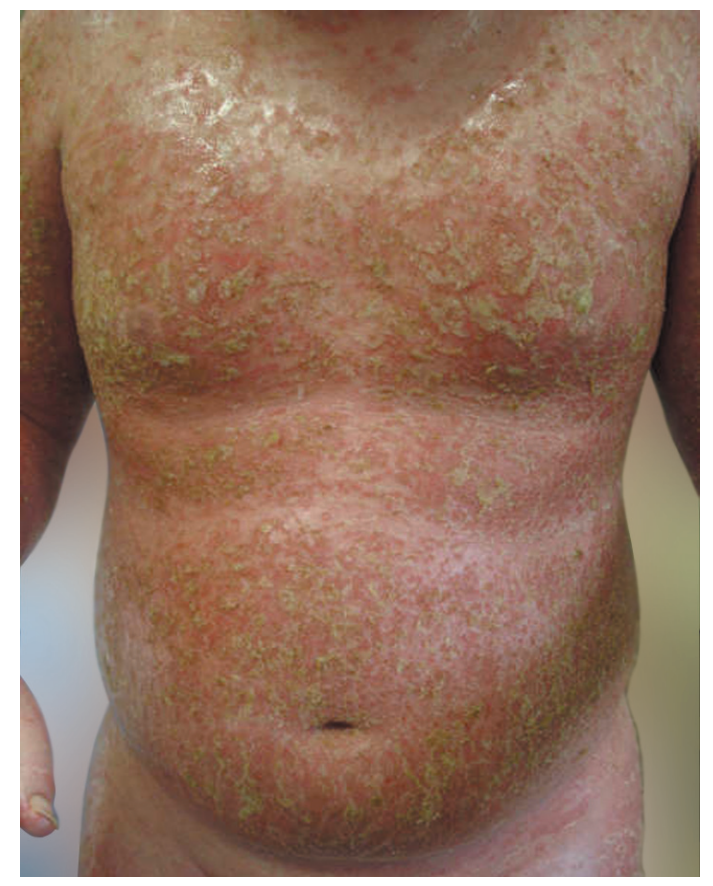

Figura 6: Psoríase eritrodérmica em menino de 11 anos de idade

depende da localização do processo psoriásico no aparelho ungueal, da intensidade e do tempo de evolução da moléstia. O aspecto mais frequente é de depressões cupuliformes, também designados de pits ungueais (unhas em dedal), por acometimento da prega ungueal proximal. Onicólise, hiperqueratose subungueal e "manchas de óleo" são outras alterações evidenciadas e correspondem ao acometimento do leito ungueal. Por fim, o acometimento da matriz ungueal pode levar à onicodistrofia e à traqueoníquia. Esses padrões tendem a ser similares em crianças e em adultos.

Acometimento acral com graus variáveis de onicodistrofia pode ser a única manifestação em crianças (Figura 8). O envolvimento ungueal aumenta com a idade, com a duração e a extensão da doença e com a presença de artrite psoriásica. ${ }^{25}$ Para confirmar o diagnóstico, é necessário sempre excluir a onicomicose por exame micológico. É preciso lembrar que ambas podem coexistir, uma vez que a psoríase ungueal aumenta a chance de contaminação por dermatófitos. ${ }^{26,27}$

\section{DIAGNÓSTICO}

O diagnóstico da psoríase é eminentemente clínico. Por meio da curetagem metódica de Brocq, obtêm-se dois achados clínicos típicos dessa dermatose: sinal da vela (estratificação das escamas) e o sinal do orvalho sangrante ou sinal de Auspitz (pequenos pontos de sangramento quando a escama é removida). ${ }^{18} \mathrm{O}$ halo ou anel de Woronoff (zona clara perilesional) é bastante característico da enfermidade, porém, raramente observado. ${ }^{19}$ 


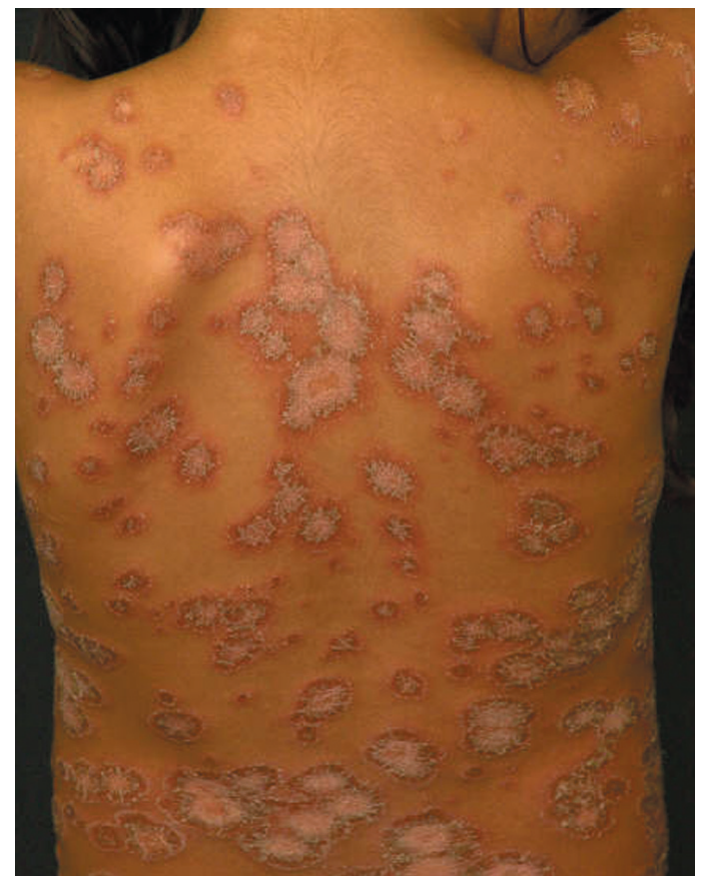

Figura 7: Forma anular da infância

O fenômeno isomórfico de Köbner manifesta o surgimento da dermatose em áreas de pele sã após diferentes tipos de trauma local em doentes acometidos pela doença e geneticamente predispostos. A psoríase caracteriza o exemplo clássico do fenômeno de Köbner, ocorrendo em cerca de $1 / 3$ dos doentes com psoríase. As lesões surgem entre 10 e 14 dias após o trauma. No entanto, o surgimento de lesões após poucos dias ou mesmo após anos também é relatado. A patogênese desse fenômeno permanece controversa, enfocando, principalmente, alterações imunológicas e vasculares. O fenômeno pode ser evidenciado em $50 \%$ das crianças com psoríase e em 39\% dos adultos acometidos. Doentes Köbner-positivos podem se tornar Köbner-

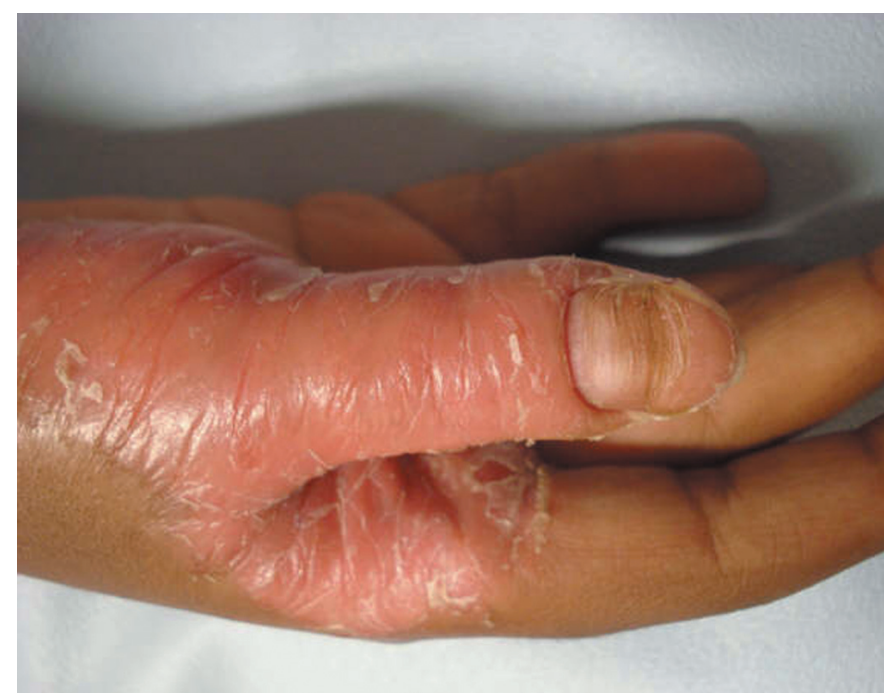

Figura 8: Psoríase acral com onicodistrofia

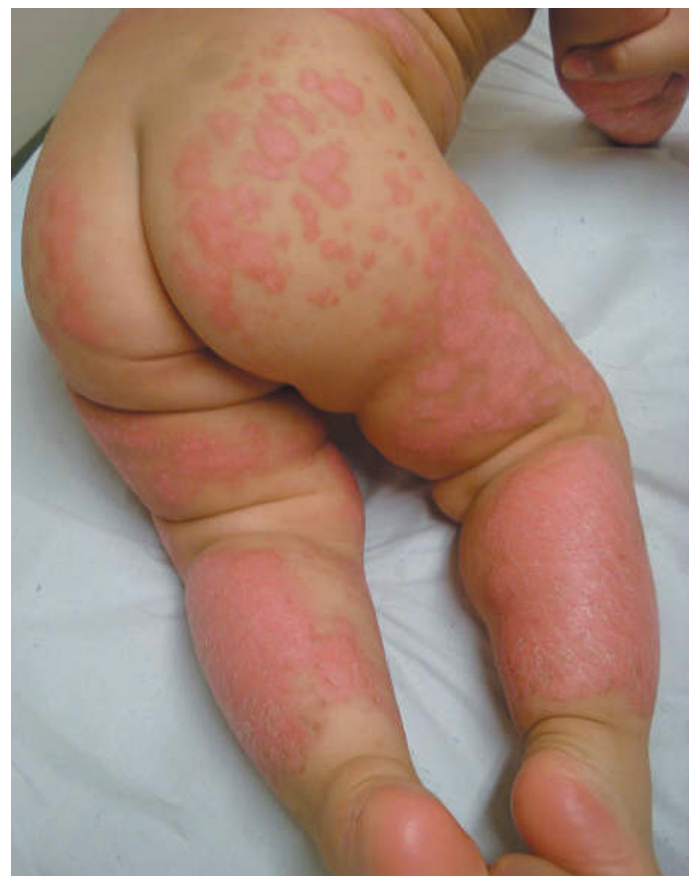

Figura 9: Psoríase em placas generalizada em criança de dois anos de idade

negativos e vice-versa, independentemente de quaisquer medidas terapêuticas empregadas. ${ }^{16,17}$

Outro fenômeno recentemente descrito, o de Renbök, também designado Köbner reverso, expressa a situação na qual o traumatismo local imposto a uma placa de psoríase resulta no desaparecimento da lesão e no surgimento de pele aparentemente sã no local. Doentes Köbner-positivos classicamente não apresentam o fenômeno de Renbök; os dois eventos parecem mutuamente exclusivos. ${ }^{20}$

Não existe exame laboratorial específico para o diagnóstico de psoríase1. O quadro histológico não é específico, porém é bastante sugestivo. As primeiras alterações evidenciadas são a vasodilatação e o infiltrado inflamatório perivascular. Este invade a epiderme, onde surge discreta espongiose, invasão de neutrófilos e paraqueratose. Em uma lesão definida, ocorre alongamento regular dos cones epiteliais, com afinamento na porção suprapapilar; as papilas estão alargadas e edemaciadas, exibindo capilares dilatados e tortuosos. Na epiderme, ocorre paraqueratose, desaparecimento da camada granular e presença de agrupamentos de neutrófilos (microabscessos de Munro). Pode acontecer, principalmente na psoríase pustulosa, a presença de cavidades contendo neutrófilos, denominadas pústulas espongiformes de Kogoj. O infiltrado inflamatório presente é discreto e composto de células mononucleares, especialmente, linfócitos. ${ }^{5,28}$

Diagnósticos diferenciais a serem considerados na infância e na adolescência incluem: dermatite seborreica, eczemas, micoses superficiais, sífilis secundária, pitiríase rubra pilar, líquen plano, lúpus eritematoso, pitiría- 
se liquenóide crônica, Nevil, acrodermatite enteropática, pênfigo foliáceo eritrodérmico, eritrodermia por drogas, pustulose subcórnea de Sneddon-Wilkinson, pustulose exantemática aguda generalizada e impetigo bolhoso (Quadro 1). ${ }^{16,19}$

\section{TRATAMENTO}

O tratamento da psoríase tem por objetivo o controle da enfermidade e a melhora da qualidade de vida do doente. Para se determinar o melhor esquema terapêutico, deve-se considerar sexo, idade, quadro clínico, gravidade da doença, sinais e sintomas associados, comorbidades, medicações concomitantes, tratamentos prévios, efeitos adversos ocorridos e a participação dos pais ou responsáveis no tratamento. Inicialmente, devem-se esclarecer os doentes e os pais ou responsáveis sobre as características da enfermidade e o seu curso, bem como orientá-los sobre a importância da exposição solar2. Para alguns doentes, o acompanhamento psicoterápico pode ser necessário. ${ }^{21}$

$\mathrm{Na}$ maioria dos doentes pediátricos, a psoríase pode ser tratada com medicações tópicas. A fototerapia é opção para casos mais extensos e refratários. A terapia sistêmica é reservada para casos graves e extensos (Figura 9), sem controle com tratamento tópico e/ou fototerapia. ${ }^{1,6,19}$ Sendo assim, as terapêuticas específicas dependerão da forma e da extensão da doença. ${ }^{28.32}$

\section{Tratamento Tópico}

$\mathrm{Na}$ forma de monoterapia ou esquema combinado, o uso de medicações tópicas costuma ser suficiente para o controle das formas leves da psoríase. Nas formas moderadas a graves, o tratamento local, quando associado à fototerapia e/ou à terapêutica sistêmica, propicia maior conforto ao doente, acelera a melhora e minimiza o prurido.

Emolientes e/ou umectantes (lactato de amônia, vaselina, ceramidas ou óleo mineral) e, nas lesões hiperqueratósicas, os ceratolíticos (ácido salicílico - 3-6\%, uréia -5-20\%) deverão ser incluídos em qualquer esquema terapêutico, seja como coadjuvantes ou em alternância com os produtos ativos ou, até mesmo, em fases assintomáticas. As opções de uso tópico são as que se seguem.

Corticosteróides tópicos: apresentam ação anti-inflamatória, antiproliferativa (antimitótica), imunossupressora, vasoconstritora e antipruriginosa. É a terapêutica tópica mais utilizada nos casos de psoríase infantil. A eficácia da resposta aos corticosteróides tópicos varia conforme a forma clínica, sendo alta na psoríase invertida, moderada na psoríase do corpo e discreta na psoríase palmo-plantar e ungueal. A localização das lesões de psoríase determina a potência dos corticosteróides tópicos a serem utilizados, devido ao risco de efeitos adversos. Corticosteróides de média e alta potências são indicados para lesões no couro cabeludo, no tronco e nos membros. Já os de menor potên- cia são indicados em lesões localizadas na face, nas regiões periauriculares, em dobras e na genitália. Após a melhora clínica, deve-se tentar a substituição por corticosteróides de menor potência, a fim de evitar o desenvolvimento de atrofia, estrias e hipertricose e a inibição do eixo hipotálamo-hipófise-adrenal, principalmente em crianças. A taquifilaxia, ou seja, a perda de eficácia pelo uso contínuo da medicação e pela necessidade de preparados cada vez mais potentes, é uma constante nesta enfermidade.

Coaltar (2-10\%): tem como veículo vaselina, cold cream ou pomadas. Quando em uso isolado, tem ação moderada na psoríase em placas, mas, quando associado à fototerapia, sua ação é potencializada. Pode ser combinado com ácido salicílico 2-5\% em lesões hiperqueratósicas. Representa opção terapêutica muito eficaz e de baixíssimo custo. No couro cabeludo, pode ser usado sob a forma de liquor carbonis detergens (coaltar 20\% em álcool $95^{\circ}$, emulsificado com extrato de quilaia, diluído em cremes ou emulsões) ou mesmo na forma de xampus. A foliculite é o efeito colateral mais frequente da utilização do coaltar. Entre os inconvenientes do seu uso, pode-se citar a baixa aceitação cosmética. Existe conflito acerca da potencial carcinogenicidade do coaltar. Apesar de estudos in vitro e estudos em animais demonstrarem claramente seu potencial carcinogênico, estudos epidemiológicos com o uso de coaltar em seres humanos não demonstram aumento da incidência de neoplasias no grupo estudado. ${ }^{33}$

Método Göckerman: indicado para psoríase disseminada em placas, não eritrodérmica. É a associação do coaltar com a radiação UVB. A pomada de coaltar é aplicada no doente, devendo permanecer pelo maior tempo possível. A aplicação de UVB é feita em doses crescentes, diariamente ou em dias alternados, sem a remoção da pomada. Após a irradiação, tomar banho para retirar escamas e reaplicar a pomada. Total de 20-30 aplicações até o clareamento das lesões.

Antralina (ou ditranol): acredita-se que seu efeito seja citostático, reduzindo a atividade mitótica das células epidérmicas psoriásicas. Pode ser usada em baixas concentrações $(0,1-0,5 \%)$ durante 24 horas ou em altas concentrações (1-3\%) em aplicações de apenas 15 a 30 minutos. Preparado em creme, pasta ou pomada. O clareamento costuma ocorrer em três a quatro semanas. Substância irritante, deve ser evitada em áreas intertriginosas, próxima aos olhos e mucosas e em pele sã perilesional, onde pode determinar erosões e bolhas. Mancha roupas, azulejos e a pele ao redor das lesões. Praticamente, não há risco de toxicidade sistêmica, apresentando excelente perfil de segurança na faixa pediátrica. Considerada medicação altamente eficaz em psoríase, leva a períodos de remissão prolongados na ausência de taquifilaxia. ${ }^{34}$

Calcipotriol: análogo da vitamina D3 que diminui a proliferação e induz diferenciação dos queratinócitos, 
QUADRo 1: Formas clínicas e principais diagnósticos diferenciais da psoríase na infância e na adolescência

\section{Forma clínica}

Psoríase em placas

Psoríase no couro cabeludo

Psoríase linear

Psoríase na área da fralda

Psoríase nas flexuras

Psoríase gutata

Psoríase pustulosa (generalizada)

Psoríase pustulosa (localizada)

Psoríase pustulosa (palmoplantar)

Psoríase ungueal

\section{Diagnósticos diferenciais}

Eczema numular

Tinea corporis

Dermatite seborreica

LESA

Tinea capitis

Dermatite atópica

Dermatite seborreica

Nevil

Blaschkitis

Acrodermatite enteropática

Dermatite de contato

Candidíase

Eritrasma

Candidíase

Eritrasma

Tinea corporis

Dermatite de contato

Líquen plano

Pitiríase rósea

Pitiríase rubra pilar

Pitiríase liquenóide crônica

Sífilis secundária

Pustulose exantemática generalizada aguda Síndrome da pele escaldada estafilocócica Dermatose pustulosa subcórnea

Tinea corporis

Eritema anular centrífugo

Síndrome de Sweet

Disidrose infectada

Tinea manum

Onicomicose

Onicodistrofia

Líquen plano além de modificar a resposta imune. É seguro e, em monoterapia, tem eficácia média para o tratamento de ataque das formas leves e moderadas de psoríase em adultos. Quando usado em esquemas combinados ou seqüenciais com corticoterapia tópica, permite períodos de remissão mais prolongados, sem o efeito "rebote" que a monoterapia com corticosteróides induz. Deve ser aplicado à noite e lavado de manhã. A eficácia e a segu- rança do calcipotriol no tratamento de pacientes pediátricos ainda não estão bem estabelecidas. Em diferentes relatos da literatura, o calcipotriol pomada tem se mostrado efetivo, bem tolerado e seguro em crianças com psoríase, sendo a irritação local o efeito colateral mais comumente relatado. ${ }^{35-38}$ Embora não existam guidelines formais para uso em crianças, o uso de até $45 \mathrm{~g} / \mathrm{sem} / \mathrm{m}^{2}$ em crianças parece não influenciar os níveis séricos de 
cálcio. $^{36}$ Pode provocar irritação da pele, especialmente da face, onde deve ser evitado. Além de prurido, eritema e ardor, podem ocorrer foliculite e alterações da pigmentação nos locais da aplicação.

Imunomoduladores tópicos: pimecrolimus e tacrolimus podem ser indicados para formas localizadas na face, dobras e semimucosas, por provocarem menos efeitos colaterais do que os corticosteróides e análogos da vitamina $\mathrm{D}$ e por apresentarem melhor absorção nessas áreas. A sua eficácia é extremamente variável. No Brasil, o pimecrolimus é indicado em crianças a partir de três meses de vida e o tacrolimus, a partir de dois anos. Não devem ser utilizados em presença de infecções viróticas, bacterianas ou fúngicas. ${ }^{39}$

Retinóides tópicos: o retinóide usado em doentes com psoríase é o tazaroteno, disponível em gel com concentração a $0,01 \%$ e $0,05 \%$. De eficácia leve a moderada, o tazaroteno é indicado para psoríase crônica em placas. Não está aprovado para uso em crianças com psoríase, porém há indicação para seu uso em acne para crianças maiores de 12 anos. Não há estudos referentes à eficácia e à segurança do seu uso em crianças com psoríase. Pode determinar irritação, queimação ou eritema local. Não deve ser usado na face ou em dobras. Não determina taquifilaxia e, atualmente, não se encontra disponível no Brasil. ${ }^{34}$

\section{Fototerapia e Tratamento Sistêmico}

Fototerapia com PUVA, UVB banda larga (290$320 \mathrm{~nm})$ e banda estreita $(311 \mathrm{~nm})$. Trata-se de opção terapêutica utilizada de modo isolado ou combinado a outras modalidades terapêuticas, tópicas ou sistêmicas. O mecanismo de ação da fototerapia se faz mediante a atividade antiproliferativa, anti-inflamatória e imunossupressora. Diferentes formas de psoríase podem ser tratadas com esse método, porém, a melhor indicação é psoríase moderada, com predomínio de placas finas. Doentes com psoríase pustulosa ou eritrodérmica não devem ser submetidos a fototerapia nem a banhos de sol, devido ao risco de piora do quadro e de vasodilatação. Em crianças, o tratamento deve ser reservado para as que possam compreender e aceitar essa modalidade terapêutica. A frequência necessária para um tratamento satisfatório é de três vezes por semana.

A radiação UVB é bastante eficaz para o tratamento da psoríase em placas e gutata. É utilizada isoladamente ou associada ao uso de coaltar (método Göckerman). O efeito colateral mais comum é a queimadura, sendo baixo o risco de câncer de pele. As contraindicações para o método são fotossensibilidade e antecedentes de melanoma. Óculos de proteção devem ser usados durante a exposição. O efeito antipsoriásico é superior quando utilizado na faixa de $311 \mathrm{~nm}$, permitindo menor tempo de exposição nessa modalidade de banda estreita. Efeitos satisfatórios ocorrem, geralmente, após oito semanas de tratamento.
O método PUVA tende a ser mais eficaz e rápido em induzir melhora quando comparado ao UVB. É associado ao 8-MOP sistêmico, tópico ou adicionado a banhos. A medicação sistêmica deve ser ingerida cerca de duas horas antes do banho de luz e apresenta o inconveniente de exigir proteção ocular por 24 horas. A dose preconizada é de $20 \mathrm{mg}$ abaixo de $50 \mathrm{~kg}$ de peso, $30 \mathrm{mg}$ entre $51 \mathrm{~kg}$ e $65 \mathrm{~kg}, 40 \mathrm{mg}$ entre $66 \mathrm{~kg}$ e $80 \mathrm{~kg}$ e $50 \mathrm{mg}$ acima de 80kg. Não há estudos demonstrando a segurança de PUVA oral em crianças abaixo de oito anos de idade, mas o método pode ser empregado em adolescentes. ${ }^{2,40,41}$

Antibióticos: embora não haja evidências de que a antibioticoterapia altere a evolução natural da psoríase gutata desencadeada por infecção, crianças com tal forma da doença e infecção estreptocócica documentada devem receber penicilina ou eritromicina por sete a 14 dias. ${ }^{1}$

Metotrexato (ametopterina): antagonista do ácido fólico, com o qual apresenta similaridade estrutural. Pode ser administrado por via oral, intramuscular e endovenosa, sendo excretado essencialmente por via renal. A biodisponibilidade da medicação diminui com a ingestão de certos alimentos, especialmente derivados do leite; no entanto, a droga não precisa ser ingerida em jejum. $O$ metotrexato deve ser empregado em casos extensos e resistentes de psoríase na infância ou em casos de psoríase artropática, eritrodérmica e pustulosa generalizada. A dose utilizada para doentes pediátricos é de 0,2$0,4 \mathrm{mg} / \mathrm{kg} / \mathrm{semana}$, até a dose total semanal de 12,5-20mg. Pode ser associado ao ácido fólico (1-5mg per os /dia). Apresenta rápido início de ação. A criança mais jovem tratada na literatura tinha quatro anos de idade e psoríase grave desde os dois anos de idade. Recentemente, tem-se associado o metotrexato, em baixas doses, ao uso de biológicos, especialmente o infliximabe, baseado na sua ação inibitória na produção de anticorpos. ${ }^{42,43}$ Essa associação não foi, até o momento, avaliada na população pediátrica. Controles hematológicos e provas periódicas das funções hepática e renal são indispensáveis. Clinicamente, um dos sinais mais precoces de intolerância é o aparecimento de lesões aftóides na mucosa oral, traduzindo leucopenia importante. O efeito colateral mais comum é a intolerância gástrica. Apresenta múltiplas interações medicamentosas. As contraindicações absolutas são gestação e lactação, cirrose hepática, infecção hepática ativa e insuficiência hepática. ${ }^{44,45}$ Vacinas de vírus vivos ou atenuados devem ser evitadas.

Acitretina: derivado da vitamina A (retinol), é empregado na dose $0,5-1,0 \mathrm{mg} / \mathrm{kg} / \mathrm{d}$. Especialmente indicado na psoríase pustulosa generalizada, também utilizada na psoríase em placas generalizadas e na psoríase eritrodérmica (ocasião em que se espera melhora após três a quatro meses). Representa a opção terapêutica sistêmica mais utilizada em crianças com quadros extensos $\mathrm{e}$ rebeldes ao tratamento tópico e à fototerapia. Os efeitos colaterais incluem: queilite leve (dose-dependente), epistaxe, conjuntivite, paroníquia, alopecia, prurido, dislipi- 
demia e teratogenia (o etretinato persiste no organismo por dois anos, devendo ser, portanto, contraindicado em mulheres em idade fértil). A terapia prolongada com acitretina deve ser avaliada com cuidado em crianças, pois há relatos de fechamento prematuro das epífises ósseas, calcificações de tendões e ligamentos e retardo do crescimento ósseo. Exames radiológicos devem ser realizados anualmente. A eficácia da acitretina tende a ser moderada, sendo alta quando associada à fototerapia. A resposta clínica é demorada. Entre as contraindicações absolutas, estão gestação ou desejo de engravidar nos próximos anos, insuficiência hepática e renal e alergia ao parabeno contido nas cápsulas. ${ }^{46}$ Não é contraindicado o uso concomitante de vacinas próprias da faixa etária.

Ciclosporina A: atua inibindo os linfócitos TCD4 ativados, impedindo a liberação de IL2. Embora tenha sido muito estudada em doentes com dermatite atópica, não há estudos de segurança e eficácia suficientes para psoríase em crianças. Deve ser reservada para casos graves, como psoríase eritrodérmica, e para casos rapidamente progressivos e sem resposta a outros métodos terapêuticos. A dose da ciclosporina é $2-5 \mathrm{mg} / \mathrm{kg}$, diariamente, por três a quatro meses, quando deve ser gradualmente retirada. Recorrências tendem a ocorrer com a diminuição da dose. Os efeitos colaterais incluem nefrotoxicidade, hipertensão, náusea, sensações parestésicas, hiperplasia gengival, hipertricose e aumento do risco de neoplasias, porém não parecem mais frequentes em crianças quando comparados a adultos com psoríase. A medicação requer monitorização renal, hematológica e hepática a cada duas a quatro semanas. As contraindicações ao uso de ciclosporina são anormalidades na função renal, hipertensão arterial sistêmica não controlada, malignidades e lactação. A imunização com vírus vivos ou atenuados deve ser evitada durante o período de tratamento e entre três e 12 meses após o seu término, dependendo da dose empregada. ${ }^{2}$ A ciclosporina apresenta múltiplas interações medicamentosas, porém, é um dos poucos tratamentos para psoríase que pode, eventualmente, ser utilizado em gestantes. ${ }^{46}$

Imunobiológicos: os imunobiológicos ou, simplesmente, biológicos representam grupo de medica- mentos que interferem de maneira específica e pontual com o sistema imune. Atuam bloqueando ou estimulando uma ou mais vias da resposta imunológica. Apesar de sua alta complexidade e variabilidade estrutural, todos os biológicos representam proteínas obtidas por meio de modernas técnicas de biotecnologia. $\mathrm{O}$ alvo desses agentes terapêuticos inclui o tráfego dos linfócitos da microcirculação para a pele, a apresentação antigênica das células apresentadoras de antígeno aos linfócitos e, por fim, as diferentes citocinas. ${ }^{6,47,48}$ São medicações de altíssimo custo. Até o momento, o etanercepte é o biológico mais criteriosamente avaliado para o uso em crianças com psoríase. Representa a forma solúvel de um receptor de fator de necrose tumoral (TNF) totalmente humano. ${ }^{49,50,51}$ A medicação recebeu sua primeira aprovação em 1998 para artrite reumatóide moderada a grave, tendo sido posteriormente (1999) aprovada para o tratamento de crianças e adolescentes portadores de artrite reumatóide juvenil. O etanercepte foi aprovado em 2004 para o tratamento de adultos portadores de psoríase em placa moderada a grave. ${ }^{52.54} \mathrm{Em}$ recente estudo da literatura, crianças entre quatro e 17 anos com psoríase moderada a grave responderam de forma favorável à medicação na dose de $0,8 \mathrm{mg} / \mathrm{kg} / \mathrm{semana}$ (máximo de $50 \mathrm{mg}$ ), administrada por via subcutânea, no total de 48 semanas, com ocorrência de quatro eventos adversos sérios (incluindo pneumonia, gastroenterite, desidratação e remoção cirúrgica de cisto ovariano) que se resolveram sem sequelas. ${ }^{55}$ A medicação aguarda aprovação na Europa e nos Estados Unidos para o uso em crianças com psoríase moderada a grave.

\section{PROGNÓSTICO}

A maioria das crianças acometidas apresenta a forma leve de psoríase com resposta favorável aos tratamentos tópicos. Embora a regressão do quadro possa ser seguida de remissão prolongada, um curso crônico e recorrente é a evolução mais comum. Em muitos casos, ocorre mudança no padrão da psoríase. Algumas crianças pioram com a idade, necessitando tratamentos mais agressivos. ${ }^{56}$ Os doentes de psoríase gutata tendem a evoluir para remissão da doença ou mesmo para psoríase em placas. ${ }^{15,26}$ 


\section{REFERÊNCIAS}

1. Hogan A. Papulosquamous disease. In: Schachner LA, Hansen RC. Pediatric Dermatology. 3rd ed. Edinburgh: Mosby; 2003. p. 643-6.

2. Consenso Brasileiro de Psoríase e guias de tratamento. Rio de Janeiro: Sociedade Brasileira de Dermatologia; 2006.

3. Watson W, Cann HM, Farber EM, Nall ML. The genetics of psoriasis. Arch Dermatol. 1972;105:197-207.

4. Burden AD. Management of psoriasis in childhood. Clin Exp Dermatol. 1999;24:341-5.

5. Carneiro SCS. Psoríase: mecanismos de doença e implicações terapêuticas [tese]. São Paulo: Faculdade de Medicina da Universidade de São Paulo; 2007.

6. Benoit S, Hamm H. Childhood psoriasis. Clin Dermatol. 2007;25:555-62.

7. Ghoreschi K, Weigert C, Röcken M. Immunopathogenesis and role of $\mathrm{T}$ cells in psoriasis. Clin Dermatol. 2007;25:574-80.

8. Sabat R, Philipp S, Höfflich C, Kreutzer S, Wallace E, Asadullah K, et al. Immunopathogenesis of psoriasis. Exp Dermatol. 2007;16:779-98.

9. Büchau AS, Gallo RL. Innate immunity and antimicrobial defense systems in psoriasis. Clin Dermatol. 2007;25:616-24.

10. Cassia FF, Carneiro SC, Marques MTQ, Pontes LF, Filgueira AL, Porto LCS. Psoriasis vulgaris and human leukocyte antigens. J Eur Acad Dermatol Venereol. 2007;21:303-10.

11. Valdimarsson $\mathrm{H}$. The genetic basis of psoriasis. Clin Dermatol. 2007;25:563-7.

12. Fry L, Baker BS. Triggering psoriasis: the role of infections and medications. Clin Dermatol. 2007;25:606-15.

13. Ockenfels HM. Triggermechanismen der Psoriasis. Hautarzt. 2003;54:215-23.

14. Gudjonsson JE, Elder JT. Psoriasis: epidemiology. Clin Dermatol. 2007;25:535-46.

15. Christophers E, Mrowietz U. Psoriasis. In: Wolff K, Goldsmith LA, Katz SI, Gilchrest BA, Paller AS, Leffell DJ. Fitzpatrick's - Dermatology in general medicine. 6th ed. New York: McGraw Hill; 2003. p. 407-27.

16. Griffiths CEM, Camp RDR, Barker JNWN. Psoriasis. In: Burns T, Breathnach S, Cox N, Griffiths C. Rook's Textbook of Dermatology. 7th ed. Oxford: Blackwell Publishing, 2004. p. 35.1- 69.

17. Janniger CK, Schwartz RA, Musumeci ML, Tedeschi A, Mirona B, Micali G. Infantile psoriasis. Cutis. 2005;76:173-7.

18. Sampaio SAP, Rivitti EA. Dermatologia. 3 ed. São Paulo: Artes Médicas; 2007. p. 231- 41.

19. Van der Kerkhof P, Schalkwijk J. Psoriasis. In: Bolognia JL, Jorizzo JL, Rapini RP. Dermatology. 2nd ed. New York: Mosby; 2008.

20. Criado PR, Valente NY, Michalany N, Martins JE, Romiti R, Aoki V, et al. An unusual association between scalp psoriasis and alopecia areata: the Renbök phenomenon. Clin Exp Dermatol. 2007;32:320-1.

21. Lewkowicz D, Gottlieb AB. Pediatric psoriasis and psoriatic arthritis. Dermatol Ther. 2004;17:364- 75.

22. Gladman DD, Brockbank J. Psoriatic arthritis. Expert Opin Investig Drugs. 2000;9:1511-22.

23. Kleinert S, Feuchtenberger M, Kneitz C, Tony HP. Psoriatic arthritis: clinical spectrum and diagnostic procedures. Clin Dermatol. 2007;25:519-23.

24. Robertson DM, Cabral DA, Malleson PN, Petty RE. Juvenile psoriatic arthritis: followup and evaluation of diagnostic criteria. J Rheumatol. 1996;23:166-70.

25. Jiaravuthisan MM, Sasseville D, Vender RB, Murphy F, Muhn CY. Psoriasis of the nail: anatomy, pathology, clinical presentation, and a review of the literature on the therapy. J Am Acad Dermatol. 2007;57:1- 27.

26. Naldi L, Gambini D. The clinical spectrum of psoriasis. Clin Dermatol. 2007;25:510-18.

27. Griffi CEM, Barker JNWN. Pathogenesis and clinical features of psoriasis. Lancet. 2007; 370:263- 71.

28. Murphy M, Kerr P, Grant-Kels JM. The histopathologic spectrum of psoriasis. Clin Dermatol. 2007;25:524-8.

29. Lowes MA, Bowcock AM, Krueger JG. Pathogenesis and therapy of psoriasis. Nature. 2007;445:866-73.

30. Menter A, Griffi CEM. Current and future management of psoriasis. Lancet. 2007;370:273-84.

31. Menter A, Gottlieb A, Feldman SR, Van Voorhees AS, Leonardi CL, Gordon KB, et al. Guidelines of care for the management of psoriasis and psoriatic arthritis: Section 1. Overview of psoriasis and guidelines of care for the treatment of psoriasis with biologics. J Am Acad Dermatol. 2008; 58:826-50.

32. Ceovic R, Pasic A, Lipozencic J, Murat-Susic S, Skerlev M, Husar K, et al. Treatment of childhood psoriasis. Acta Dermatovenerol Croat. 2006;14:261-4.

33. Pion IA, Koenig KL, Lim HW. Is dermatologic usage of coal tar carcinogenic? A review of the literature. Dermatol Surg. 1995;21:227-31.

34. Cordoro KM. Topical therapy for the management of childhood psoriasis: part I. Skin Therapy Lett. 2008;13:1-3.

35. Choi YJ, Hann SK, Chang SN, Park WH. Infantile psoriasis: successful treatment with topical calcipotriol. Pediatr Dermatol. 2000;17:242-4.

36. Darley CR, Cunliffe WJ, Green CM, Hutchinson PE, Klaber MR, Downes N. Safety and efficacy of calcipotriol ointment (Dovonex) in treating children with psoriasis vulgaris. Br J Dermatol. 1996;135:390-3.

37. Oranje AP, Marcoux D, Svensson A, Hutchinson PE, Klaber MR, Downes N. Topical calcipotriol in childhood psoriasis. J Am Acad Dermatol. 1997;36(2 Pt 1):203-8.

38. Travis LB, Silverberg NB. Psoriasis in infancy: therapy with calcipotriene ointment. Cutis. 2001;68:341-4.

39. Cordoro KM. Systemic and light therapies for the management of childhood psoriasis: part II. Skin Therapy Lett. 2008; 13:1-3.

40. Wolff K. Side-effects of psoralen photochemotherapy (PUVA). Br J Dermatol. 1990;122 Suppl 36:117-25.

41. MacDonald A, Burden AD. Psoriasis: advances in pathophysiology and management. Postgrad Med J. 2007;83:690-7.

42. Hersh EM, Carbone PP, Wong VG, Freireich EJ.. Inhibition of primary immune response in man by antimetabolites. Cancer Res. 1965;25:1997-2001.

43. Mitchell MS, Wade ME, DeCenti RC, Bertino JR, Calabresi P. Immune suppressive effects of cytosine arabinoside and methotrexate in man. Ann Intern Med. 1969;70:535-47.

44. Gladman DD, Mease PJ, Krueger G, Van der Heidje DM, Antoni C, Helliwell PS, et al. Outcome measures in psoriatic arthritis. J Rheumatol. 2005;32:2262-9.

45. Gottlieb A, Korman NJ, Gordon KB, Feldman SR, Lebwohl $\mathrm{M}$, Koo JY, et al. Guidelines of care for the management of 
psoriasis and psoriatic arthritis: Section 2. Psoriatic arthritis: overview and guidelines of care for treatment with an emphasis on the biologics. J Am Acad Dermatol. 2008; 58:851-64.

46. Consenso Brasileiro de Psoríase e Guias de Tratamento. Rio de Janeiro: Sociedade Brasileira de Dermatologia; 2006.

47. Chong BF, Wong HK. Immunobiologics in the treatment of psoriasis. Clin Immunol. 2007;123:129-38.

48. Smith CH, Anstey AV, Barker JNWN, Burden AD, Chalmers RJ, Chandler D, et al. British Association of Dermatologists: guidelines for use of biological interventions in psoriasis 2005. Br J Dermatol. 2005;153:486-97.

49. Goffe B, Cather JC. Etanercept: an overview. J Am Acad Dermatol. 2003; 49(Suppl):S105-11.

50. Gottlieb AB. Etanercept for the treatment of psoriasis and psoriatic arthritis. Dermatol Ther. 2004;17:401-8

51. Tan JK, Alphale A, Malaviya R, Sun Y, Gottlieb AB. Mechanisms of action of etanercept in psoriasis. J Investig Dermatol Symp Proc. 2007;12:38-45.

52. Tyring S, Gottlieb A, Papp K. Etanercept and clinical outcomes, fatigue, and depression in psoriasis: double-blind placebo-controlled randomized phase III trial. Lancet. 2006; 367:29-35.

53. Leonardi CL, Powers JL, Matheson RT, Goffe BS, Zitnik R, Wang A, et al. Etanercept as monotherapy in patients with psoriasis. N Engl J Med. 2003;349:2014-22.

54. Wu EQ, Feldman SR, Chen L, Kaltenboeck A, Yu AP, Gupta SR, et al. Utilization pattern of etanercept and its cost implications in moderate to severe psoriasis in a managed care population. Curr Med Res Opin. 2008;24:3493-501.

55. Paller AS, Siegfried EC, Langley RG, Gottlieb AB, Pariser D, Landells I, et al. Etanercept treatment for children and adolescents with plaque psoriasis. $\mathrm{N}$ Engl J Med. 2008;358:241-51.

56. Morris A, Rogers M, Fischer G, Arch B, Williams K. Childhood psoriasis: review of 1262 cases. Pediatr Dermatol. 2001;18:188-98.

ENDEREÇO PARA CORRESPONDÊNCIA / MAILING ADDRESS: Ricardo Romiti

Rua Dr. Neto de Araújo, 320 - Cj. 1.004-1.005

Vila Mariana

04111001 - São Paulo - SP

Tel./fax: (11) 5549-2211

E-mail:rromiti@botmail.com

Como citar este artigo/How to cite this article: Romiti R, Maragno L, Arnone M, Takahashi MDF. Psoríase na infância e na adolescência. An Bras Dermatol. 2009;84(1):9-22. 